IRA-International Journal of Technology \& Engineering ISSN 2455-4480; Vol.06, Issue 02 (2017)

Pg. no. 29-37

Institute of Research Advances

https://research-advances.org/index.php/IRAJTE

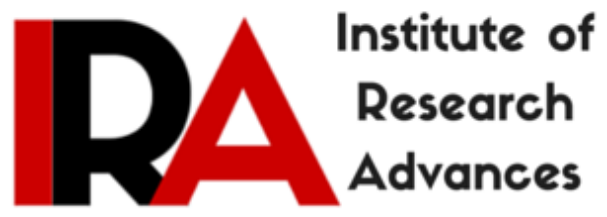

\title{
A Survey on Image Compression using Burrows Wheeler Transform
}

\author{
Manasi Rath ${ }^{1}$ Suvendu Rup ${ }^{2}$ \\ ${ }^{1,2}$ Utkal University, Vani Vihar, IIIT, Bhubaneswar, India.
}

Type of Reviewed: Peer Reviewed.

DOI: http://dx.doi.org/10.21013/jte.v6.n2.p3

\section{How to cite this paper:}

Rath, M., \& Rup, S. (2017). A Survey on Image Compression using Burrows Wheeler Transform. IRA-International Journal of Technology \& Engineering (ISSN 2455-4480), 6(2), 29-37. doi:http://dx.doi.org/10.21013/jte.v6.n2.p3

(C) Institute of Research Advances

\section{(c) BY-NC}

This work is licensed under a Creative Commons Attribution-Non Commercial 4.0 International License subject to proper citation to the publication source of the work.

Disclaimer: The scholarly papers as reviewed and published by the Institute of Research Advances (IRA) are the views and opinions of their respective authors and are not the views or opinions of the IRA. The IRA disclaims of any harm or loss caused due to the published content to any party. 


\begin{abstract}
This paper is a methodological review paper on image compression using Burrows Wheeler Transform. Normally BWT is used for text compression but it has been recently applied to image compression field. Basically it is a lossless compression technique which is used for high level resolution.This paper proposes about several scheme added with BWT to improve the performance of image compression which helps us to formulate a new technique for the further improvement in BWT. Here many authors have different type of representation of BWT for better compression.
\end{abstract}

Keywords- Image compression; Text compression; BWT; BWCA

\title{
1. Introduction
}

Now a days the amount of digital contents increases in a rapid rate and the communication and transform of data also increases gradually. But the amount of storage and the bandwidth do not increase at the same speed. Hence to overcome the problem the powerful and efficient compression techniques are required. Over the year a large number of approaches have been proposed the text compression [2]. The data compression is very important for saving expensive data storage space and for achieving a faster data transmission ratio.

The Burrows-Wheeler Transform (BWT) is a combinatorial algorithm originally created for text compression and that has been recently applied to image compression field.The BWT is a block sorting lossless data compression technique which was first time proposed by M.Burrows and D.Wheeler in 1994[1].It is widely used because of its less complexity and invertibleness. This compression technique is composed of 3 stages: The BWT itself, MTF and Entropy Coding. The first two stages are just a transform technique; The Move-To-Front (MTF) is the heart of the BWT algorithm. It is generally used for permuting a string to achieve better compression in third stage[10].

In these days it is a novel technique for image compression. In this framework if we use BWT directly into an image then result are not acceptable. In order to improve the compression performance we have to formulate an innovative method. In this paper we have a survey on the use of BWT to enhance the compression ratio and how BWT is incorporated in different step of the compression technique. For example, areas where the pictures are used for some specific information extraction, medical imaging, image archiving system, exact image analysis, remote sensing and GIS, cultural-heritage, scientific and industrial sector, surveillance.

In this paper, image compression using BWT is introduced. The rest of the paper is organized as follows; Section 2 depicts the literature review, Section 3 contains Results and discussion, Finally Section 4 provides the concluding remarks and the future work.

\section{Review of the Literature}

\section{Text Preprocessing Using EIDBE}

Several authors have represented the original algorithm in their own way. They incorporate different technique with BWT to improve the performances of the compression. Senthil and Robert had published 
in[9] about EIDBE as an encoding strategy. EIDBE is the enhancement in dictionary based coding. In this paper the conjunction of EIDBE and BWT is used to achieve better compression for text data. From the experimental result it can be analyzed that EIDBE achieve better compression at the processing stage and enough redundancy is retained and get better result.

\section{On Lossless Image Compression using the BWT}

Don and Kalyan [8] proposed two special scheme of BWT based image coding namely BLIC and BLICX which is later being based on context ordering property of BWT. Each uses neither MTF nor RLE stages in compressing the image. They have used simple MED for prediction and order-1 arithmetic coding which could improve the result. BLIC is slightly faster than standard BWT compression and BLICX takes about twice the time as BWT. Here the processing time is also reduced.

\section{Lossless Image Compression Using Kernel Based GST}

M.Asif,Aftab, M.Younus and Aasia [7] have proposed a unique method improving Global Structure Transform (GST) stage of Burrows Wheeler Compression Algorithm (BWCA) for color image. This proposed model applied the MTF transform at GST stage by 2D block of BWT data. The experimental result shows that the modification of MTF gives improved performance on an average compared to the original BWCA scheme.

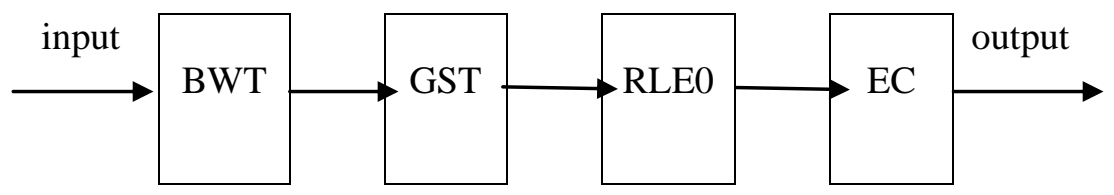

Figure 1. Typical scheme of the Burrows-Wheeler Compression Algorithm

\section{A fast and efficient post BWT-stage for Burrows-Wheeler Compression algorithm}

Abel [5] has proposed a new concept for Burrows Wheeler Compression Algorithm. He incorporated a new stage incremental frequency count (IFC) as post BWT and EC stage of algorithm. This stage gives a higher throughput as MTF stage. The main idea of IFC stage is to raise increment which is depending on the average index value of the near past. The RLE stage is many times faster than a ranking scheme. Hence it is used before the IFC stage. Hence it achieves double compression time as fast as the BWT based compression algorithm.

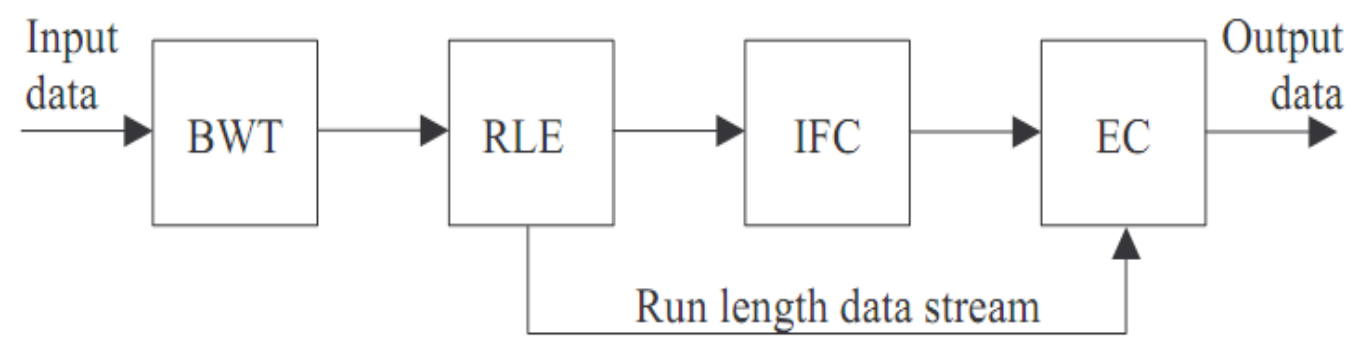

Figure 2.The BWCA with RLE and IFC stage 


\section{Universal Lossless Source Coding With the Burrows Wheeler Transform}

Michells, Karthik, Sanjeev and Sergio Verdu [3] have proposed theoretical evolution of BWT based coding in their paper. This paper includes (1) Statistical characterization of the BWT output on both finite and sequence of length $n \rightarrow \infty$. (2) A variety of very simple new techniques for BWT based lossless source coding. (3) Proofs the universality and bounds on the rates of convergence of both new and existing BWT based code for finite memory and stationary ergoidic source. They theoretically justified and experimentally concluded that BWT based coding of Universal lossless coding performance that converges to the optimal coding performance more quickly than the Ziv-Lempel style codes.

\section{Waveform and image compression using BWT and the wavelet transform}

In 1997 Haitao and C. Sidney [2] have proposed a novel waveform and image compression technique using Burrows Wheeler Transform. Here they used Discrete Wavelet Transform (DWT) before BWT and demonstrated a comparison with Embbed Zero tree Wavelet (EZW) compression for images. Further they used pre coding and arithmetic coding to achieve the level of performance.

They demonstrate that the simple concatenation of the DWT and BWT coding performs comparably as the embedded zero tree wavelet (EZW) compression for images. The wavelet transform is a powerful analysis tool, and that has been successfully used in data compression. Let's continue with a simple example. The DWT of the signal in figure 3 (a) is shown. In figure 4 (a), where Daubechie's length-6 orthogonal wavelet is used for the 6-scale transform. Here they quantize the wavelet coefficients uniformly $2^{12}$ levels, so that after the inverse wavelet transform and rounding to the nearest integer the result is lossless. As there are a large number of zeros found in the wavelet coefficients, which are trivially self-repetitive (runs of zeros are invariant under the BWT). From Figure 4 (b),BWT of the DWT coefficients not only have a large number of zeros, but also have many long runs of identical values. Figure 4 (c-d) shows the MTF outputs of (b). Clearly, more zeros have appeared.
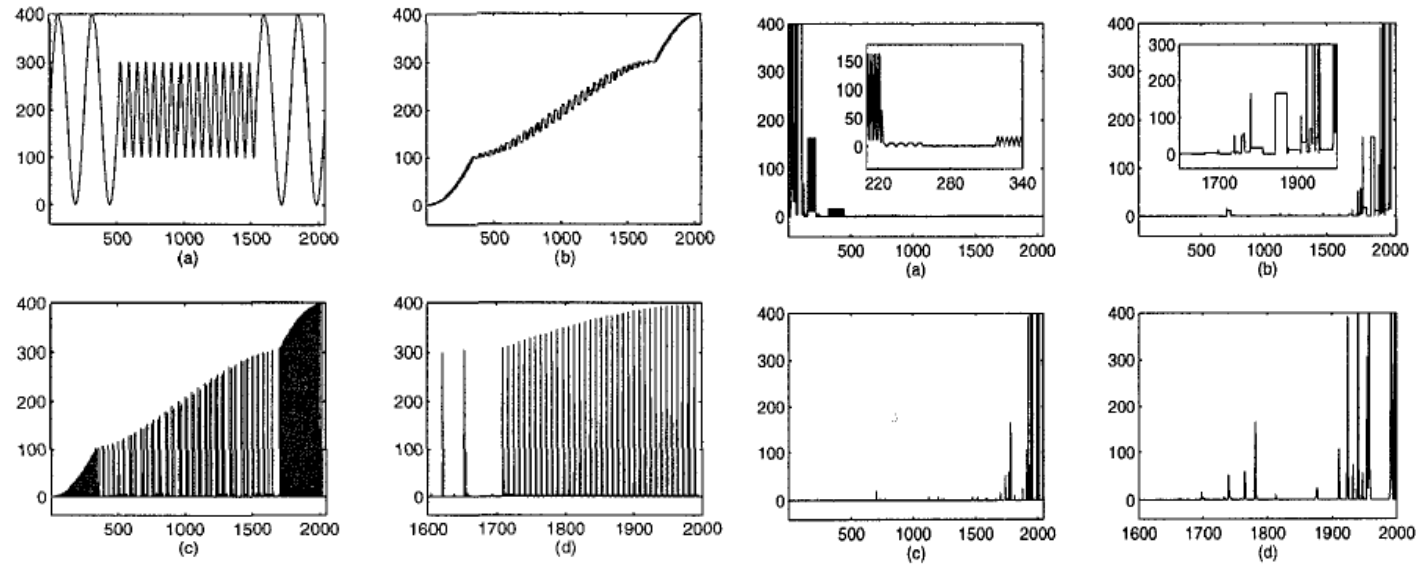

Figure3: example of coding of waveforms using the waveform coding using the

BWT. (a) The original waveform. (b) The BWT output signal in figure1(a). (b)The of (a). (c) The MTF output of (b). (d) The closeup of (c) (d) The close-up of (c).

Figure4:The continuing example of

DWT and BWT. (a) The DWT of the BWT of (a). (c) the MTF output of (b). 


\section{Colour image compression using bit reduction and BWT}

S.Annadurai and R. Shanmugalakshmi [4] have implemented BWT for color image compression. In this paper they proposed a new image compression technique for color images using bit reduction and BWT. In Bit reduction technique a block of pixel values are converted in to equivalent alphabet which gives rise to a new encoding text files. After that the BWT is applied for a reversible transformation to a block of text to make redundancy in the input. Then Hoffman coding and run length coding is used for elimination of redundancies of pixels.

The proposed Compression algorithm consists of two phases:

1) Encoding and

2) BWT Compression

Source image Text file compressed image

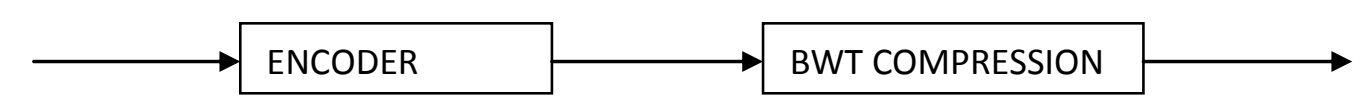

Figure 5.Block diagram of Compression Algorithm

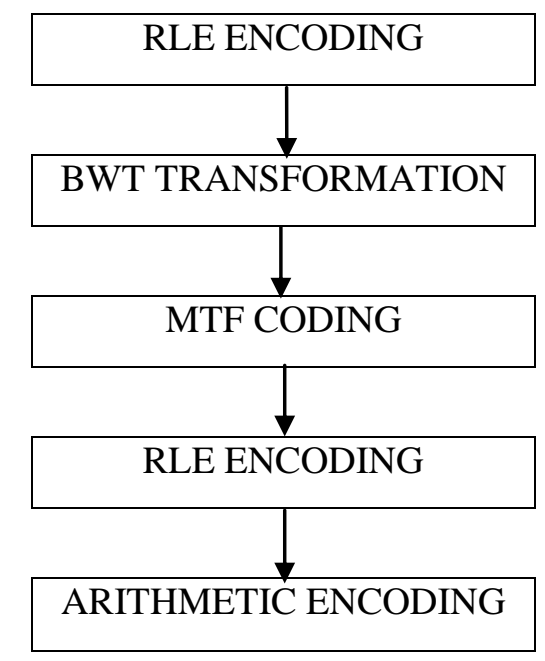

Figure 6.Stages in BWT Compression

Decompression is done in two stages:

1) BWT Decompression

2) Decoding procedure 


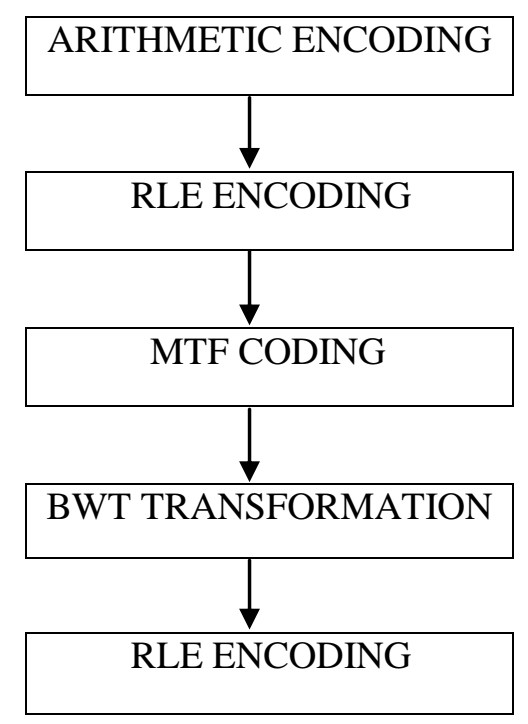

Figure 7.Stages in BWT Decompression

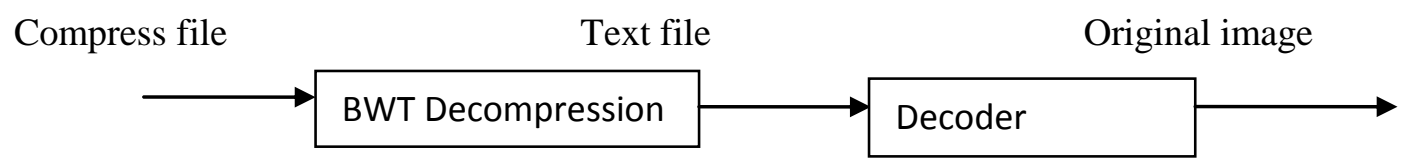

Figure 8. Block diagram of Decompression Algorithm

In this paper they also make a comparison between this approach and statistical compression technique. Here the experimental result shows that this approach has 50\% more compression rate than the conventional approach.

\section{Burrows-Wheeler Transform based JPEG}

Yair [6] has proposed a paper on Burrows Wheeler based JPEG compression. In that paper he suggested a replacement of traditional Hoffman coding by Burrows wheeler compression. There are two reasons for using the JPEG algorithm-first, it reduces size of image files and second is it stores full colour information.

STEPS:-

- Transform the image colour into a suitable colour space.

- Apply DCT.

- Perform the quantization. 
- Instead of assigning Huffman codes to each DC value and each pair of zeros' length and an AC value assign a number to each of them.

- $\quad$ Process the above numbers by BWT.

- $\quad$ Process the output by Move-to-front procedure and Run length encoding.

- Compress by Arithmetic encoding (for fixed length).

Here the replacement yields the better compression ratio in case of high quality image. It gives better performance in case of synthetic and poor quality image. But the main Burrows Wheeler based JPEG has long compression time.

\section{Results and Discussion}

The baseline image compression algorithm using the DWT and BWT is a very efficient technique. Here the images are first wavelet transformed and then quantized. The bit-rate V.S. PSNR plot for the Lena image is shown. Here the simple scheme performs well in comparison with the embedded zero tree wavelet compression. Many improvements have been made on the baseline system. Various 2D to 1D scan methods are tested and result in slight decrease of bit rate at the same quality level. Another major improvement comes from a variation of the coding method. Instead of coding the quantized coefficients directly using the BWT, here the number of bits required to represent the coefficients, and then separately code the actual bits for non-zero coefficients only. This results in another 0.3 to $0.5 \mathrm{~dB}$ gain in PSNR [2].

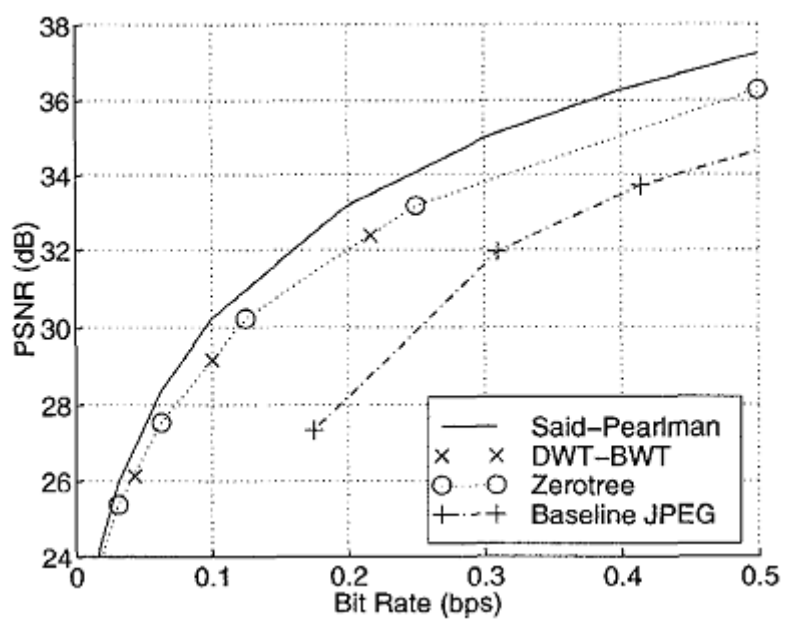

Figure 9.Performance of the baseline image compression algorithm using the DWT and BWT for the Lena image. (x) For DWT-BWT based method. (0) for the embedded zero tree wavelet compression. 


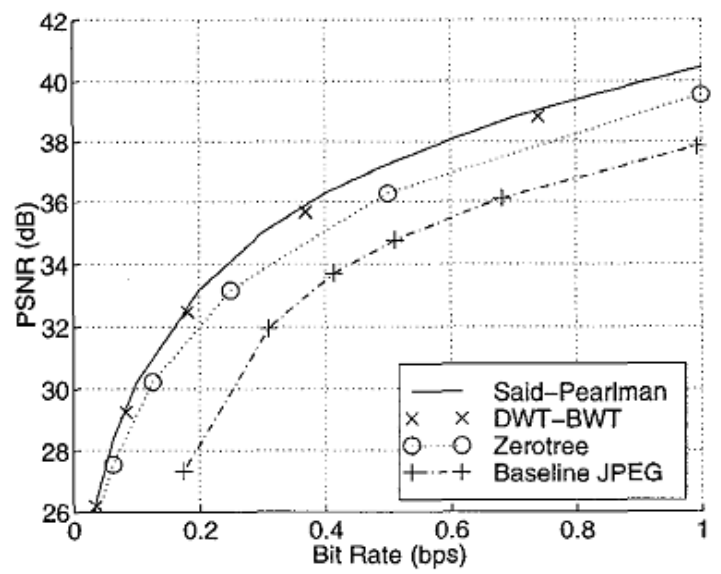

Figure 10.Performance of improved image compression algorithm using the DWT and BWT for the Lena image. (x) For improved DWT-BWT based method. (0) for the embedded zero tree wavelet compression.

In color images using bit reduction and BWT the redundancies of the pixels are eliminated in two different steps. Here the conventional approaches like Huffman coding and Run Length Coding results compression ratios only 5 to 6 . From the experimentation it has been found that the quality of the final decompressed image is exactly the same as that of the original image.

Table 1 Experimental Results

\begin{tabular}{|l|l|l|l|}
\hline File Name & $\begin{array}{l}\text { Original } \\
\text { size(bytes) }\end{array}$ & $\begin{array}{l}\text { Proposed } \\
\text { method(bytes) }\end{array}$ & $\begin{array}{l}\text { Compression } \\
\text { ratio (\%) }\end{array}$ \\
\hline $\begin{array}{l}\text { Golden } \\
\text { Hill.bmp }\end{array}$ & 49,102 & 45,261 & 8 \\
\hline Lena.bmp & 196,608 & 192,512 & 2.1 \\
\hline Boat.bmp & 44,339 & 41,062 & 7.4 \\
\hline $\begin{array}{l}\text { Mandrilla. } \\
\text { bmp }\end{array}$ & 196,608 & 175,104 & 10.9 \\
\hline
\end{tabular}




\section{Conclusions and Future work}

BWT is generally used for text compression but now these days it is a novel technique for image compression. The Burrows wheeler algorithm will achieve better compression standard that significantly improve the performance on efficiency. Though there are number of compression methods available, the need for improve performance demands newer and better techniques. Hence we are aiming to extend our research by formulating other efficient techniques based on BWT. We want to develop an improved version of BWT and realizing its efficiency in near future.

\section{References}

[1]M. Burrows and D.J. Wheeler, “A Block-sorting Lossless Data Compression Algorithm”, SRC Research Report May 10, 1994

[2]Haitao Guo and C. Sidney Bums, "Waveform and Image Compression Using the Burrows Wheeler Transform and the Wavelet Transform”, 0-8186-8183-7/97 IEEE 1997

[3]Michelle Effros, Karthik Visweswariah,Sanjeev R. Kulkarni and Sergio Verdu, "Universal Lossless Source Coding With the Burrows Wheeler Transform",IEEE TRANSACTIONS ON INFORMATION THEORY, VOL. 48, NO. 5, MAY 2002

[4]S.Annadurai and R. Shanmugalakshmi, "Color Image Compression Using Bit Reduction And Burrows Wheeler Transform”,Image and Video Coding, TENCON2003

[5]Juergen Abel, "A fast and efficient post BWT-stage for Burrows-Wheeler Compression algorithm", Proceedings of Data Compression Conference (DCC'05) 1068-0314/05, IEEE2005

[6]Yair Wiseman, “Burrows-Wheeler Based JPEG”, Data Science Journal, Volume 6, 5 March 2007

[7]M. Asif Ali, Aftab Khan, M.Younus Javed and Aasia Khanum, "Lossless Image Compression Using Kernel Based Global Structure Transform (GST)",978-1-4244-8058-6/10, 6th International Conference on Emerging Technologies (ICET), IEEE 2010

[8]Don Adjeroh and Kalyan V. Bhupathiraju, "On Lossless Image Compression using the BurrowsWheeler Transform”, 978-1-4577-1303-3/11,18th International Conference on Image Processing,IEEE 2011

[9]Senthil Shanmugasundaramand Robert Lourdusamy, "Text Preprocessing Using Enhanced Intelligent Dictionary Based Encoding (EIDBE)”,978-1-4244-8679-3/11, IEEE2011

[10] K. S. Ng and L. M. Cheng, "Lossless Image Compression By Using Gradient Adjusted Prediction And Burrows-Wheeler Transformation,",IEEE Transactions on Consumer Electronics, Vol. 45, No. 2, MAY 1999 